\title{
Влияние на длительность пикосекундного стимулированного излучения GaAs разогрева носителей заряда этим излучением
}

\author{
(c) Н.Н. Агеева, И.Л. Броневой , Д.Н. Забегаев, А.Н. Кривоносов \\ Институт радиотехники и электроники им. В.А. Котельникова Российской академии наук, \\ 125009 Москва, Россия \\ ฯE-mail: bil@cplire.ru
}

Поступила в Редакцию 19 августа 2020 г.

В окончательной редакции 30 августа 2020 г.

Принята к публикации 9 сентября 2020 г.

\begin{abstract}
Сравниваются измеренные в реальном времени огибающие мощной пикосекундной оптической накачки и собственного стимулированного пикосекундного излучения тонкого слоя GaAs. Объяснение превышения длительности излучения над длительностью накачки базируется на отрицательной обратной связи между интенсивностью излучения и разогревом носителей этим излучением. Главным параметром указанного превышения длительности является характерное время охлаждения носителей заряда, замедляемое из-за разогрева носителей излучением. Отмечается влияние этого времени и на перенормировку запрещенной зоны из-за кулоновского взаимодействия носителей. Та часть представления о пикосекундной динамике интенсивного стимулированного излучения GaAs, которая получена к настоящему моменту в наших с соавторами работах, дана в Заключении.
\end{abstract}

Ключевые слова: стимулированное пикосекундное излучение, длительность пикосекундного излучения, разогрев носителей заряда излучением, время охлаждения носителей заряда, энергетический транспорт носителей заряда, перенормировка запрещенной зоны, пикосекундная динамика излучения.

DOI: $10.21883 / F T P .2021 .02 .50495 .9510$

\section{1. Введение}

Ранее на основании экспериментально выявленной пикосекундной эволюции спектров просветления (увеличения прозрачности) тонкого слоя GaAs было предположено, что в этом слое во время пикосекундной оптической накачки носителей заряда, создававшей просветление, возникает интенсивное пикосекундное стимулированное излучение. Это предположение позволило обнаружить и объяснить затем значительное число новых нелинейных оптоэлектронных эффектов, вызванных таким излучением. Вслед за экспериментальными исследованиями характеристик излучения, интегрального по времени, были измерены в реальном времени огибающие спектральных мод излучения [1] и огибающая излучения, интегрального по спектру, которое далее для краткости будем называть $s$-излучение. В работе [2] было экспериментально обнаружено, что характерное время $\tau_{r}$ релаксации $s$-излучения и просветления связано с характерным временем охлаждения носителей заряда $\tau_{T}$. Аналитическое выражение для времени $\tau_{T}$, учитывающее влияние разогрева носителей излучением, было получено в работе [3] и обсуждается в разд. 3. В работе [4] была обнаружена антикорреляция между временем $\tau_{T}$ и интенсивностью $s$-излучения в его максимуме. Следовательно, время $\tau_{T}$ влияло не только на релаксацию, но и на возрастание $s$-излучения. Экспериментальные результаты этих двух работ соответствовали предположению, что энергетический транспорт носителей в направлении экстремумов зон проводимости и валентной, откуда носители рекомбинируют, определяет интенсивность $s$-излучения, и время $\tau_{T}$ характеризует этот транспорт. Закон Бугера в этой ситуации, как было обнаружено в работе [4], неприменим к $s$-излучению.

Настоящая работа, продолжающая названные выше исследования, посвящена изучению отличия длительности $s$-излучения от длительности накачки и связям этого с $\tau_{T}$, а значит, с указанным нагревом носителей. В ней показано, что обнаруженные: (а) запаздывание максимума $s$-излучения от максимума накачки; (б) еще большее запаздывание начала экспоненциальной релаксации $s$-излучения, существенно увеличивающее длительность $s$-излучения; (в) итоговое превышение длительности $s$-излучения над длительностью накачки и т.д., - все описывается с помощью времени $\tau_{T}$, и по сути вызвано замедлением энергетического транспорта носителей заряда из-за их нагрева. Отмечается влияние разогрева носителей и на перенормировку запрещенной зоны, возникающую из-за кулоновского взаимодействия носителей. В прикладном аспекте, изучавшееся явление указывает способ сокращения длительности излучения. В настоящей работе этим методом длительность излучения изменялась вдвое.

Поясним, что нынешняя работа во многом базируется на наших многолетних взаимосвязанных исследованиях пикосекундной динамики стимулированного излучения GaAs. Поэтому приводимые ссылки на них, без которых ряд утверждений выглядел бы бездоказательным и не проверяемым, являются вынужденными, а не саморекламой. Заменить эти ссылки на работы других авторов не удается, возможно, вследствие специфичности спектрофотохронометрического комплекса, на котором велись исследования. 


\section{2. Экспериментальные результаты}

Огибающие импульсов (изменение интенсивности со временем) накачки $I_{\mathrm{ex}}(t)$ и $s$-излучения $I_{s}(t)$ измерялись в реальном времени. Измерения проводились при комнатной температуре $T_{R}$ на пикосекундном лазерном спектрофотохронометрическом комплексе. Подробное описание и схема комплекса приведены в работе [4]. Исследуемый образец представлял собой гетероструктуру $\mathrm{Al}_{x} \mathrm{Ga}_{1-x} \mathrm{As}-\mathrm{GaAs}-\mathrm{Al}_{x} \mathrm{Ga}_{1-x} \mathrm{As}$. Слои $\mathrm{Al}_{x} \mathrm{Ga}_{1-x} \mathrm{As}$ были прозрачны для света, используемого в эксперименте. На поверхности образца, кроме торцевых, было нанесено антиотражающее покрытие, чтобы не было вертикального резонатора. Накачка слоя GaAs осуществлялась световым импульсом длительностью $(F W H M) T_{\mathrm{ex}} \approx 10$ пс. Результаты измерений указанных огибающих, представленные в графическом виде, будем называть хронограммами. Измерения выполнялись при варьируемых в одних случаях, а в других - фиксированных диаметрах $D$ и плотностях энергии импульса накачки $W_{\mathrm{ex}} / S$, где $W_{\mathrm{ex}}$ - энергия импульса накачки, $S$ - площадь сечения луча накачки. Энергия фотона накачки была $\hbar \omega_{\mathrm{ex}}=1.558$ эВ. При измерении огибающей стимулированного излучения регистрировалась часть излучения, выходившая из накачанной области слоя $\mathrm{GaAs}$ и распространявшаяся внутри телесного угла $1.2 \cdot 10^{-2}$ стерадиан с осью, ортогональной плоскости гетероструктуры. Это объясняется следующим. Распространение света в веществе должно сопровождаться рассеянием этого света из-за наличия в реальных средах оптических неоднородностей. Указанная часть излучения была выбрана для измерений потому, что выходит прямо

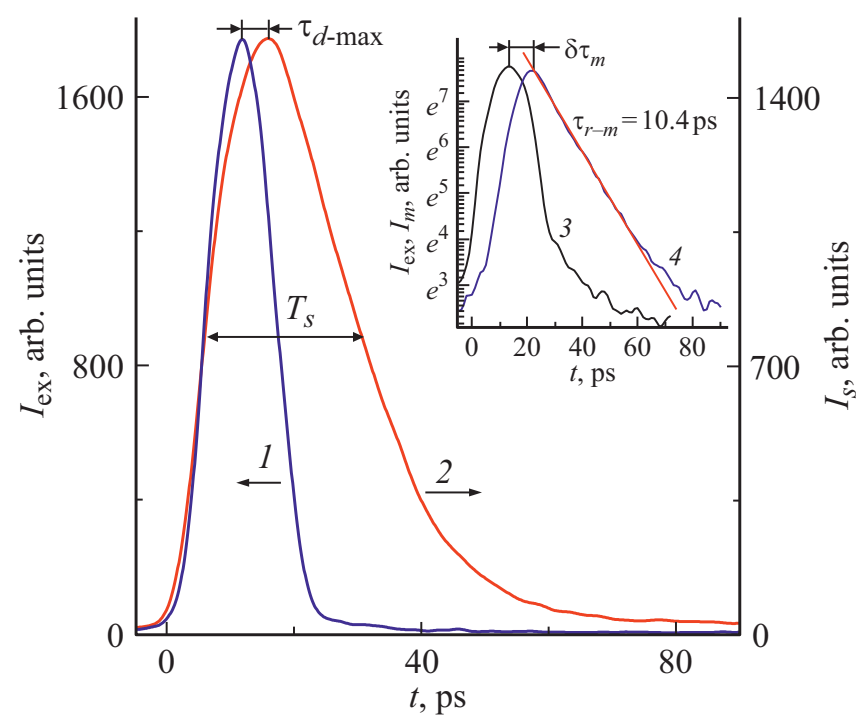

Рис. 1. Хронограмммы импульса накачки $I_{\text {ex }}(t)(1)$ и $s$-излучения $I_{s}(t)$ (2) при диаметре луча накачки $D=0.54$ мм. На вставке показаны, в полулогарифмическом масштабе, хронограммы накачки (3) и спектральной моды с энергией фотона $\hbar \omega_{m}=1.401$ эВ из работы [5] (4). К спаду хронограммы моды проведена касательная, справа от которой указано характерное время ее релаксации $\tau_{r-m}$.

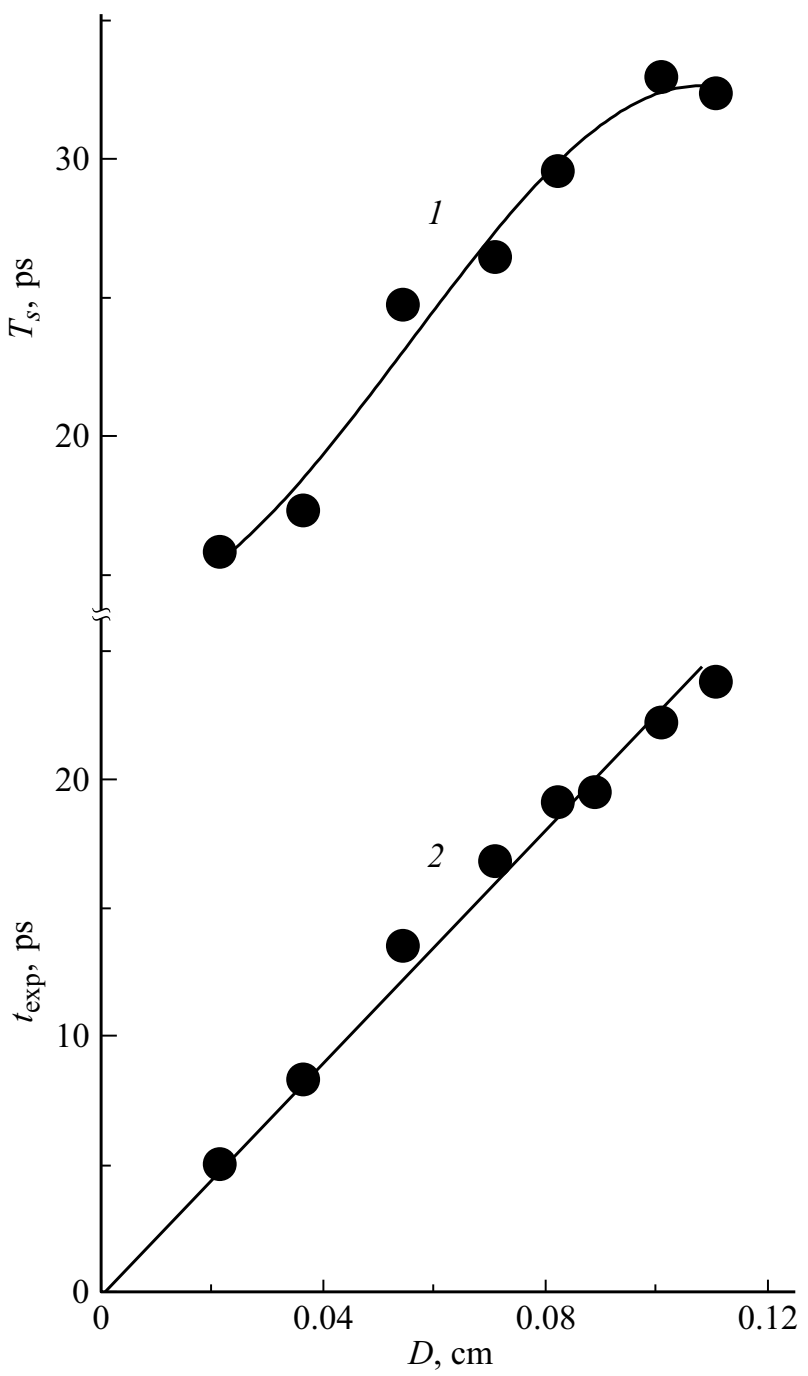

Рис. 2. Длительность импульса $s$-излучения $T_{s}(F W H M)(1)$ и интервал времени $t_{\text {exp }}(2)$ в функции от диаметра $D$ при $W_{\mathrm{ex}} / D^{2}=$ const.

из активной области, не изменяясь из-за поглощения в пассивной области и отражения от торцов образца. То, что излучение, как распространяющееся вдоль эпитаксиального слоя, так и вытекающее через поверхность гетероструктуры из-за несовершенства последней, это одно и то же стимулированное излучение, доказано в наших предыдущих исследованиях. Доказательства суммированы в работе [2]. В эксперименте при фиксированных $W_{\text {ex }} / S$ и $T_{\text {ex }}$, при изменении диаметра в интервале $D=0.02-0.11$ см были измерены описываемые далее характерные параметры огибающей $s$-излучения, определяющие ее отличие от огибающей накачки.

На рис. 1 представлены хронограммы накачки и $s$-излучения, измеренные при $D=0.54 \mathrm{мм}$. Видно, что длительность $s$-излучения на полувысоте $(F W H M) T_{s}$ существенно больше $T_{\text {ex }}$, а максимум излучения достигается позднее, чем максимум накачки. Будем обозначать время подобной задержки $\tau_{d \text {-max }}$, как это показано на том же рисунке. На большей части увеличения диаметра $D$ 
возрастали и $T_{s}$, и $\tau_{d \text {-max }}$, а к концу исследуемого интервала $D$ они начинали слегка уменьшаться (рис. 2,3). Максимально достигнутая при фиксированном диамет-

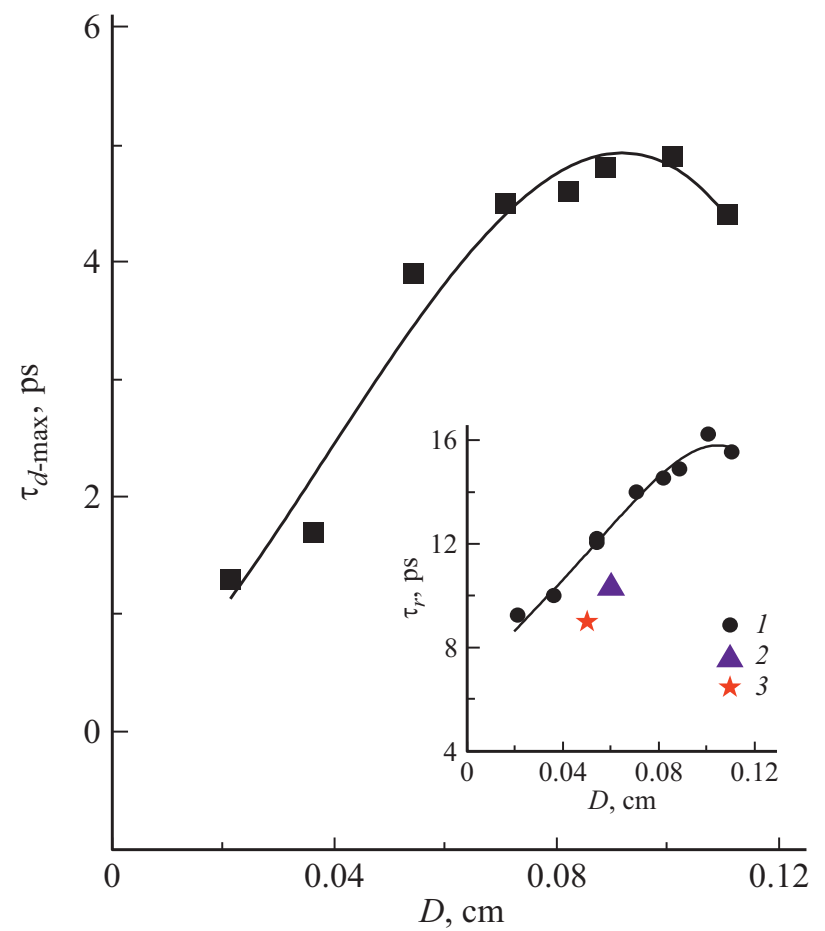

Рис. 3. Зависимость от диаметра $D$ задержки максимума $s$-излучения $\tau_{d \text {-max }}$ относительно максимума накачки. На вставке - экспериментальные значения характерного времени релаксации $s$-излучения $\tau_{r}$ в функции $D: 1-$ при $W_{\mathrm{ex}} / D^{2}=$ const, 2 - из работы [5], 3 - из работы [6]. Сплошные линии проведены для наглядности.

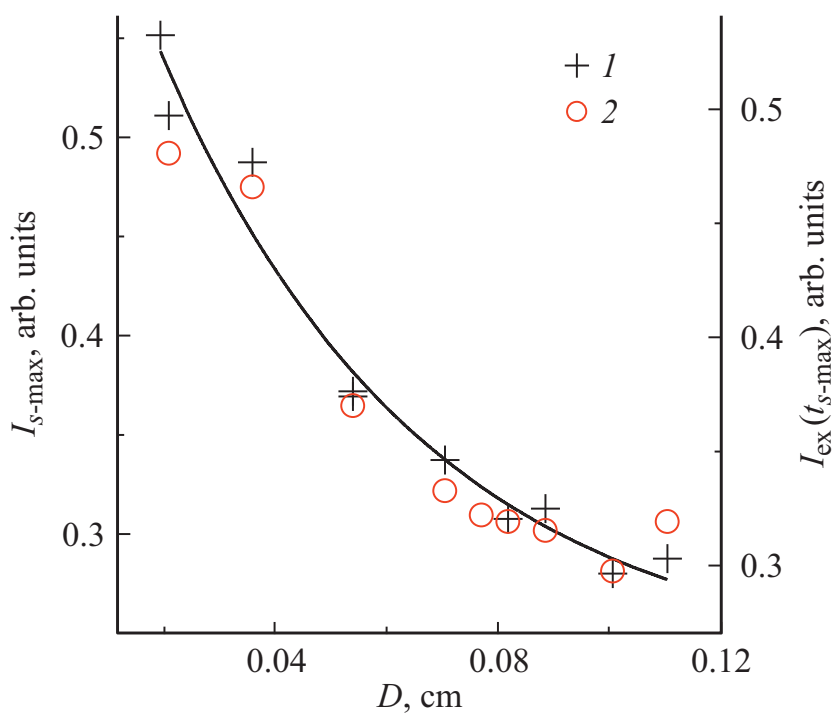

Рис. 4. Зависимость от диаметра $D$ максимальной интенсивности $s$-излучения $I_{s-\max }(1)$ и интенсивности света накачки $I_{\mathrm{ex}}\left(t_{s-\max }\right)(2)$ в тот момент $t_{s-\max }$, когда интенсивность $s$-излучения достигала максимума. Сплошная линия проведена для наглядности.

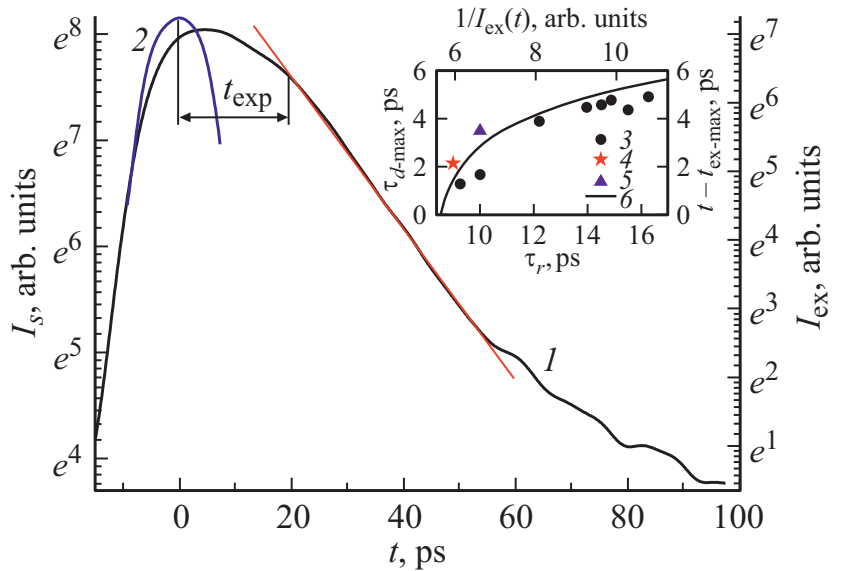

Рис. 5. Хронограмма $s$-излучения $(1)$ и вершина хронограммы накачки (2) при $D=0.82$ мм. Прямая, касательная к спаду хронограммы $s$-излучения, показывает участок экспоненциальной релаксации излучения, $t_{\text {exp }}$ - интервал времени между моментом максимальной интенсивности накачки и началом экспоненциальной релаксации. На вставке показано время $\tau_{d \text {-max }}$ в зависимости от $\tau_{r}: 3-$ при $W_{\mathrm{ex}} / D^{2}=$ const, $4-$ из работы [6], 5 - из работы [5], кривая 6 - график зависимости $t-t_{\mathrm{ex}-\max }=f\left(1 / I_{\mathrm{ex}}\right)($ см. текст статьи).

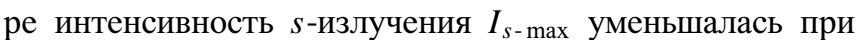
увеличении $D$, как это было обнаружено в работе [4], (рис. 4). Интенсивность света накачки $I_{\mathrm{ex}}\left(t_{s-\max }\right)$ в тот момент $t_{s-\max }$ на ее спаде, когда интенсивность $s$-излучения достигала максимума $I_{s \text {-max }}$, тоже уменьшалась при увеличении диаметра $D$, что иллюстрирует тот же рисунок. Выявляется подобие зависимостей $I_{\text {ex }}\left(t_{s-\max }\right)=f(D)$ и $I_{s-\max }=f(D)$.

В течение времени $t_{\exp }$ с того момента, как накачка достигнет максимума, и до того, когда $s$-излучение начинает экспоненциально релаксировать с характерным временем $\tau_{r}$, интенсивность $s$-излучения спадает относительно медленно. На рис. 5, где хронограммы даны в полулогарифмическом масштабе, отмечены интервал времени $t_{\exp }$ и участок экспоненциальной релаксации $s$-излучения, соприкасающийся с касательной прямой линией. Время $t_{\exp }$ росло приблизительно пропорционально увеличению диаметра $D$, (рис. 2 ), и по величине $t_{\exp } \gg \tau_{d \text {-max. }}$. Релаксация со временем $\tau_{r}$ исследовалась в работе [2] и поясняется в разд. 3.

\section{3. Обсуждение результатов}

Обозначим аббревиатурой ЭТН транспорт неравновесных носителей в энергетическом пространстве на уровни в области экстремумов зон проводимости и валентной, откуда они рекомбинируют. Ранее упомянутые работы позволяют считать, что ЭТН и вынужденная рекомбинация носителей находятся в динамическом равновесии. Оно поддерживается излучением, которое авторегулирует обеднение инверсной заселенности электронов. ЭТН происходит преимущественно благодаря тому, 
что неравновесные горячие или теплые электроны отдают энергию решетке путем излучения LO-фононов [7]. Дырки должны термализовываться с электронами за субпикосекундные времена в условиях наших экспериментов, как показывают оценки, выполненные согласно данным работы [8]. ЭТН увеличивает инверсию заселенности энергетических уровней. От этого интенсивность излучения возрастает. Это возрастание интенсивности в свою очередь усиливает: (а) внутризонное поглощение носителями заряда $s$-излучения; (б) рекомбинацию „холодных“ носителей. Эти два процесса вызывают дополнительный разогрев электронов. Разогрев ведет к замедлению ЭТН и снижению инверсии населенности, а отсюда к замедлению вынужденной рекомбинации и уменьшению интенсивности излучения. Таким образом, существует отрицательная обратная связь (ООС) между температурой носителей и интенсивностью излучения, рассмотренная в работе [3].

OОC, согласно расчетам из работы [3], должна замедлить охлаждение носителей так, что характерное время охлаждения $\tau_{T}$ будет определяться выражением

$$
\tau_{T}=\left(A+B \tau_{p} T_{c}^{1 / 2} E_{g-a}\right) \tau_{h},
$$

где $A=6.3, B=0.4 ; \tau_{h} \approx 0.8$ пс - время релаксации энергии ЭДП за счет эмиссии оптических фононов с учетом разогрева последних [7], $\tau_{T}, \tau_{p}, \tau_{h}$ измеряются в пс. В нашем случае принималось: $T_{c}-$ средняя температура носителей в $\mathrm{K}, E_{g-a}-$ средняя ширина запрещенной зоны в эВ. Время $\tau_{p}$ - это характерное время движения фотона излучения в активной среде, пока фотон не покинет эту среду или не будет поглощен:

$$
\tau_{p}^{-1} \approx c \cdot \chi^{-1}\left(\gamma+D^{-1}\right) .
$$

Здесь $\chi=3.6$ - показатель преломления $\mathrm{GaAs}$, $\gamma=\sigma n-$ коэффициент внутризонного поглощения света, $\sigma=1.5 \cdot 10^{-17} \mathrm{~cm}^{2}$ [9]. Так как при интенсивном стимулированном излучении температура и плотность носителей $n$ становятся взаимосвязаны [10] и др., то характерное время релаксации плотности носителей и интенсивности излучения становится равным

$$
\tau_{r} \approx(2 / 3) \tau_{T},
$$

что неоднократно подтверждалось в наших работах экспериментально. Предполагая, что именно с ЭТН, а значит, в значительной степени с $\tau_{T}$, преимущественно связано превышение длительности $s$-излучения над длительностью накачки, мы будем сопоставлять далее экспериментальные результаты со временем $\tau_{r}$, пользуясь его прямой связью с $\tau_{T}$ (3). Это удобнее, так как $\tau_{r}$ - это реально измеряемое время. Как видно из нижеследующего, хотя $\tau_{T}$ в теории [3] должно относиться к охлаждению носителей при релаксации одномодового излучения по окончании накачки, отличие длительности $s$-излучения от длительности накачки и особенности огибающей $s$-излучения можно охарактеризовать эмпирическими зависимостями от $\tau_{r}$. С учетом (3) это означает, что в $\tau_{T}$ заключены свойства ЭТН не только во время релаксации, но и в значительной степени во время возрастания $s$-излучения.

В начале накачки первой, с разницей во времени $\sim 1$ пс, разгорается группа мод с энергией фотона $\hbar \omega_{s}>E_{g 0}$, где $E_{g 0}$ - ширина запрещенной зоны невозбужденного GaAs. Затем в процессе роста интенсивности накачки и плотности накачанных носителей происходит упомянутая выше перенормировка (сужение) запрещенной зоны. Тогда последовательно разгораются моды с энергией фотона $\hbar \omega_{s}<E_{g 0}$, генерируемые при рекомбинации носителей с образующихся при перенормировке энергетических уровней. Все эти моды не одновременно, а последовательно достигают своего максимума интенсивности [1], а потом экспоненциально релаксируют. Последнее иллюстрирует вставка на рис. 1. Интенсивность $s$-излучения определяется ЭТН, который, следовательно, в значительной степени определяет, когда $s$-излучение достигнет максимума, когда и с какой скоростью будет происходить его релаксация. В настоящее время для того, чтобы охарактеризовать ЭТН, мы располагаем аналитическим выражением (1).

На вставке к рис. 3 представлена зависимость $\tau_{r}=f(D)$, измеренная в работе [2]. Эта зависимость удовлетворительно согласуется с ее оценкой по формулам (1)-(3), сделанной в той же работе. Там же треугольником и звездочкой показаны используемые далее экспериментальные значения $\tau_{r}$ из работ [5] при $D \approx 0.06$ см и [6] при $D \approx 0.05$ см соответственно, где накачка была более интенсивной и поэтому, как и должно быть согласно (1)-(3), время $\tau_{r}$ меньше.

Обсуждая то, что и во время накачки должно происходить замедление излучения из-за указанного разогрева носителей, в работе [3] не было дано простых аналитических выражений для этого этапа, которые можно было бы сравнить с экспериментом. Такие выражения, где замедление связано с $\tau_{r}$, а по сути с $\tau_{T}$, получаются далее эмпирически, из приведенных в разд. 2 экспериментальных зависимостей, и подтверждаются данными других наших работ.

ЭТН и интенсивность $s$-излучения растут во время накачки до того момента уже на ее спаде, когда скорость накачки носителей сравнивается со скоростью ЭТН. В этот момент интенсивность $s$-излучения достигает максимума. Это доказывается в первом приближении подобием зависимостей $I_{\mathrm{ex}}\left(t_{s-\max }\right)=f(D)$ и $I_{s-\max }=f(D)$ (рис. 4). ${ }^{1}$ Тогда зависимость $I_{s-\max }=f\left(t_{s-\max }\right)$ должна быть подобна верхней части спада хронограммы накачки $I_{\mathrm{ex}}=f(t)$. Из этого, если учесть обнаруженную в [4] антикорреляцию $I_{s-\max } \sim 1 / \tau_{r}$, несложно вывести, что графически форма зависимости $\tau_{d \text {-max }}=f\left(\tau_{r}\right)$, представленной экспериментальными точками, качественно должна быть схожа с формой указанной части зависимости $t-t_{\mathrm{ex}-\max }=f\left(1 / I_{\mathrm{ex}}\right)$, что и подтверждается на вставке к рис. 5, здесь $t_{\mathrm{ex}-\max }-$ момент достижения максимума накачки. Если представленный на вставке график $t-t_{\mathrm{ex}-\max }=f\left(1 / I_{\mathrm{ex}}\right)$ описать в координатах

\footnotetext{
${ }^{1}$ Планируется посвятить этому специальное исследование.
} 


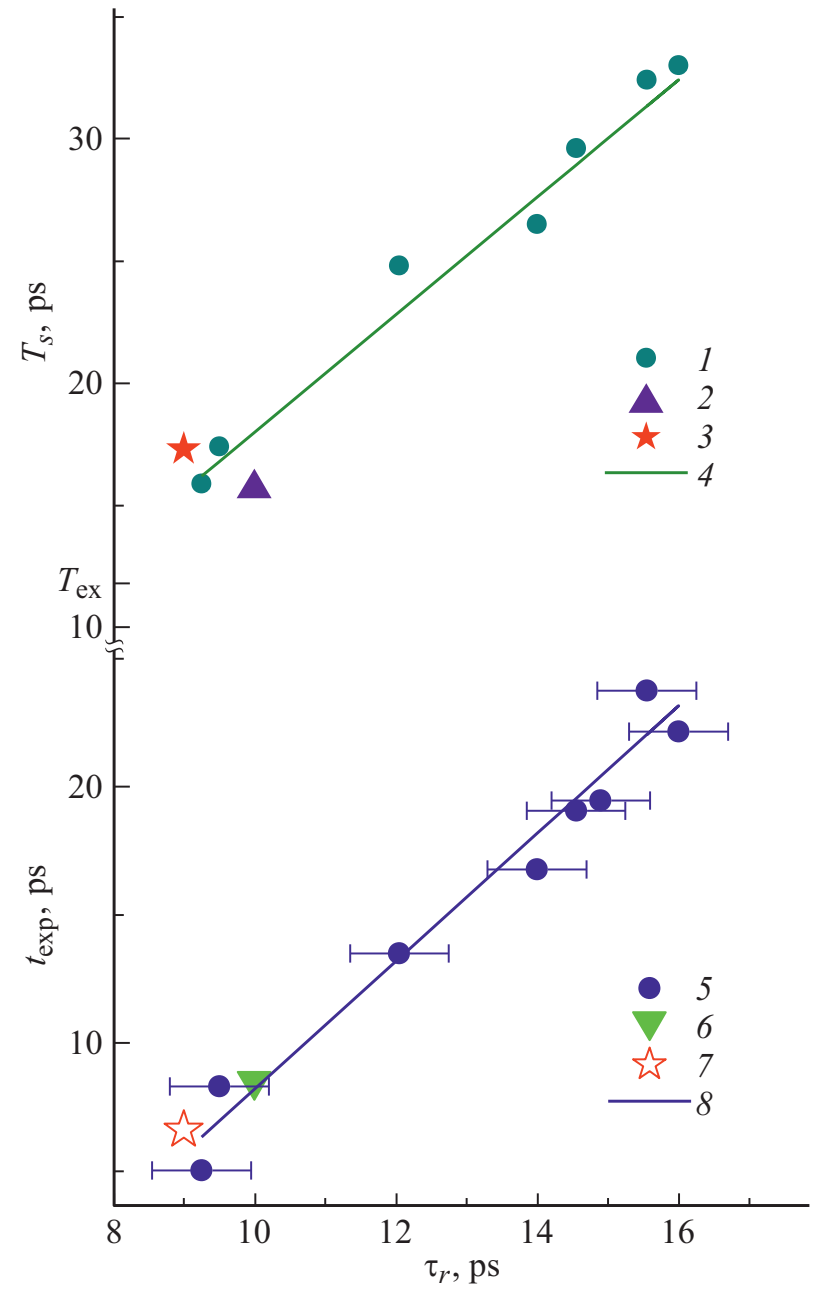

Рис. 6. Длительность импульса $s$-излучения $T_{s}$ и интервал времени $t_{\exp }$ в функции от характерного времени релаксации $s$-излучения $\tau_{r}: 1,5-$ при $W_{\text {ex }} / D^{2}=$ const; $2,6-$ из работы [5]; 3,7 - из работы [6] при $W_{\mathrm{ex}}=6.8$ отн. ед. Прямые $(4),(8)$ поясняются в тексте. $T_{\mathrm{ex}}$ на оси ординат - длительность импульса накачки $(F W H M)$.

$\tau_{d \text {-max }}, \tau_{r}$, то он будет отображать логарифмическую зависимость, как и должно быть в свете вышесказанного при квазигауссовом импульсе накачки,

$$
\tau_{d-\max } \approx 1.84+1.74 \cdot \ln \left(\tau_{r}-8.2\right),
$$

удовлетворительно согласующуюся с экспериментальными точками. Последние определяли из зависимостей $\tau_{r}=f(D)$ и $\tau_{d-\max }=f(D)$. Заметим, что небольшой разброс точек на вставке к рис. 5 может быть вызван, во-первых, тем, что там собраны результаты измерений, выполненных в разные дни и даже годы, в разных работах. Соответственно, форма огибающей накачки не была строго одинаковой при всех измерениях. Но и при

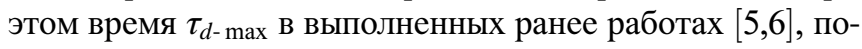
казанное звездочкой и треугольником на вставке к рис. 5 , удовлетворительно согласуется с зависимостью (4), подтверждая ее. Во-вторых, имелась и определенная по-

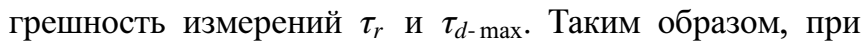

гауссовом импульсе накачки, задержка $\tau_{d \text {-max }}$ растет приблизительно логарифмически при увеличении $\tau_{r}$, как представлено выражением (4).

Согласно вышесказанному, моды не одновременно достигали максимума, и пока это продолжалось до начала экспоненциальной релаксации $s$-излучения, отделяемого от максимума излучения интервалом времени $t_{\text {exp }}$, интенсивность $s$-излучения уменьшалась „замедленно“. Как показывает график задержки $t_{\operatorname{exp~}}$ в функции $\tau_{r}$ (см. рис. 6), полученный из экспериментальных графиков $t_{\exp }, \tau_{r}=f(D)$, эту функцию можно аппроксимировать выражением

$$
t_{\exp }=2.5 \tau_{r}-16.8(\text { пс) },
$$

которому соответствует прямая 8 и с которым удовлетворительно согласуются также задержки $t_{\text {exp}}$, определенные теперь для работ $[5,6]$ и показанные на том же рисунке. Таким образом, время $t_{\text {exp }}$ растет пропорционально $\tau_{r}$ и, значит, определяется тоже нагревом носителей излучением. В работе [5] задержка $t_{\exp }=8.5$ пс для $s$-излучения была равна задержке $\delta_{t m}=8.5$ пс (см. вставку на рис. 1) максимума спектральной моды с энергией фотона $\hbar \omega_{m}=1.401$ эВ относительно максимума накачки. Характерные времена релаксации $s$-излучения $\tau_{r}=10$ пс и указанной моды $\tau_{r-m}=10.4$ пс практически совпадали. Эта мода относилась к числу самых интенсивных в спектре, и во время экспоненциальной релаксации $s$-излучения ее интенсивность значительно преобладала над интенсивностью других мод. Мода с $\hbar \omega_{m}=1.401$ эВ особенна еще и тем, что генерируется при рекомбинации электронов с одного из самых низкоэнергетичных уровней из числа тех, которые существовали до перенормировки запрещенной зоны.

Превышение длительности $s$-излучения $T_{s}$ над длительностью накачки $T_{\mathrm{ex}}-$ это суммарный продукт задержки $t_{\exp }$ и релаксации со временем $\tau_{r}$, связанных с разогревом носителей. На рис. 6 представлен график $T_{s}$ в функции $\tau_{r}$, которая аппроксимируется выражением

$$
T_{s}=2.4 \tau_{r}-6(\text { пс }) .
$$

Этому выражению соответствует прямая 4. Столь простая связь с $\tau_{r}$ подтверждает, что $T_{s}$, как и рассмотренные выше $t_{\text {exp }}$ и $\tau_{d \text {-max }},-$ все определяются вышеописанным разогревом носителей, характеризуемым временем $\tau_{T}$, связанным с $\tau_{r}$ соотношением (3). Для практического использования, по-видимому, существенно то, что превышение длительности $s$-излучения над длительностью накачки пропорционально $\tau_{r}$. Например, как видно на рис. 6 , при уменьшения $\tau_{r}$ время $T_{s}$ уменьшалось вдвое.

Так как $\tau_{r}$ для излучения изменялось преимущественно путем изменения $D$ при $W_{\text {ex }} / D^{2}=$ const, то следует подчеркнуть, что и при приблизительно одинаковом диаметре $D$ различие $\tau_{r}$ приводило к таким изменениям рассмотренных выше параметров $s$-излучения, которые соответствовали полученным аппроксимирующим зависимостям. Так, если при $D=0.54$ мм и $\tau_{r}=12.1$ пс было 
$\tau_{d \text {-max }}=3.9$ пс, $t_{\exp }=13.5$ пс, $T_{s}=24.8$ пс, то, как выводится из данных работы [6], при $D=0.5$ мм и уменьшении времени релаксации до $\tau_{r}=9$ пс аналогичные параметры уменьшались соответственно до значений $\tau_{d-\max }=2.2$ пс, $t_{\text {exp }}=6.6 п$ с, $T_{s}=17.3$ пс, (см. рис. 6 и вставку на рис. 5).

Коснемся еще раз дефицита перенормировки $E_{g}$. Дефицит возникает при увеличении интенсивности излучения, а значит, усилении ЭТН. Усиление ЭТН связано с уменьшением $\tau_{r}$. Следовательно, при уменьшении $\tau_{r}$ должен еще и возрастать дефицит перенормировки, т. е. недостаточно сужаться запрещенная зона. Сравним результаты измерений при уже приводившихся выше почти одинаковых диаметрах луча накачки, но разных $\tau_{r}$. Меньшему $\tau_{r}$ в такой ситуации должна, согласно (1)-(3), соответствовать большая плотность носителей, а значит, в отсутствие ЭТН было бы и большее сужение $E_{g}[10,11]$. При $D=0.54$ мм и $\tau_{r}=12.1$ пс была получена $\hbar \omega_{e}=1.369$ эВ, где $\hbar \omega_{e}-$ энергия фотона длинноволнового края спектра излучения, интегрального по времени, приблизительно представляющая предел, до которого сужалась во время излучения запрещенная зона. При $D=0.5$ мм и $\tau_{r}=9$ пс была $\hbar \omega_{e}=1.375$ эВ [6]. Таким образом, при меньшем значении $\tau_{r}$, а значит, при более интенсивном ЭТН, получилось большее значение $\hbar \omega_{e}$, а значит, и большее значение $E_{g}$. Это можно пояснить и таким образом. Чем больше разогрев носителей излучением, тем слабее становится ЭТН. Поэтому он меньше мешает установиться коррелированному распределению носителей в энергетическом пространстве. Дефицит перенормировки из-за этого меньше, а сама перенормировка (сужение $E_{g}$ ) больше.

Заметим, что выше мы использовали среднее экспериментальное характерное время релаксация $s$-излучения $\tau_{r}$. Однако в течение излучения взаимосвязанные параметры $E_{g}, T_{c}$ и $\tau_{p}$ изменяются, поэтому в соответствии с (1), (3) могут изменяться во времени $\tau_{r}$ и $\tau_{T}$. Этого, в принятом в настоящей работе первом приближении, мы не учитывали.

\section{4. Заключение}

В итоге установлено, что превышение длительности пикосекундного излучения, интегрального по спектру, над длительностью пикосекундной накачки, особенности формы огибающей излучения вызваны преимущественно разогревом носителей, создаваемым излучением. Разогрев замедляет энергетический транспорт накачанных электронов (ЭТН) к экстремумам зон проводимости и валентной, откуда они вынужденно рекомбинируют. Предполагается, что транспорт и рекомбинация находятся в динамическом равновесии, поддерживаемом излучением путем авторегулирования обеднения инверсной заселенности электронов. На данном этапе исследования ЭТН характеризуется временем $\tau_{T}$ - характерным временем охлаждения носителей заряда. Так как $\tau_{T}$ пропорционально $\tau_{r}$ - реально измеряемому характерному времени релаксации излучения, то исследовались зависимости от $\tau_{r}$, подразумевая, что в их основе лежит зависимость от $\tau_{T}$. Для того чтобы именно так воспринимались выводы работы, далее полученные зависимости от $\tau_{r}$ будут представляться, как зависимости от $\tau_{T}\left(\tau_{r}\right)$.

Выявлено, что временем $\tau_{T}\left(\tau_{r}\right)$ определяется длительность излучения $-T_{s}$, а также отставание от максимума накачки: (а) максимума излучения $-\tau_{d \text {-max }}$, (б) начала этапа экспоненциальной релаксации излучения $-t_{\text {exp. }}$ Время $\tau_{d-\max }$ показывает, что, когда ЭТН, растущий замедленно из-за разогрева носителей, сравнивается с накачкой носителей на ее спаде, именно в этот момент ЭТН достигает максимума. По первым наблюдениям

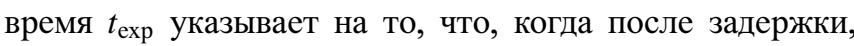
вызванной нагревом носителей излучением, ЭТН достигает максимальной интенсивности и затем начинает экспоненциально релаксировать с характерным временем $\tau_{r-m} \approx \tau_{r}$ мода, интенсивность которой доминирует на экспоненциальном спаде излучения, и которая генерируется при рекомбинации электронов с одного из самых низкоэнергетичных уровней из числа тех, которые существовали до перенормировки запрещенной зоны.

В работе [3], где было выведено выражение для $\tau_{T}$, не было дано простых, необходимых для сравнения с экспериментом, аналитических выражений, которые характеризовали бы вызываемое нагревом носителей замедление излучения на этапе его возрастания, затем до начала экспоненциальной релаксации, и уже с учетом этого и релаксации - длительность излучения. Такие аппроксимирующие экспериментальные результаты, выражения, где замедление связано с $\tau_{T}\left(\tau_{r}\right)$, получены эмпирически в настоящей работе и могут использоваться как первое приближение. Из них вытекает актуальная для приложений рекомендация, что уменьшения $T_{s}$ можно достигать, уменьшая $\tau_{T}\left(\tau_{r}\right)$. Это делается, например, путем сокращения длины активной области или увеличения накачки носителей. Таким путем, как видно из результатов данной работы, $T_{s}$ уменьшалась вдвое. В этой же работе обнаружилось, но пока в единичном сравнении, что с возрастанием $\tau_{T}\left(\tau_{r}\right)$ уменьшается дефицит перенормировки запрещенной зоны, вызываемой кулоновским взаимодействием носителей. Это говорит в пользу интерпретации образования дефицита, данной в работе [6], и несколько дополняет ее. Настоящая работа является одним из первых этапов исследования влияния разогрева носителей излучением на ЭТН и вместе с получением новой экспериментальной информации позволяет наметить темы для дальнейшего изучения этого.

Суммируя вышеописанные результаты с полученными ранее, изложим ту часть представления о пикосекундной динамике интенсивного стимулированного излучения GaAs, которая на сегодня получена в наших работах. ${ }^{2}$

\footnotetext{
${ }^{2} \mathrm{C}$ перечнем публикаций можно ознакомиться, например, в разделе http://www.cplire.ru/rus/bil/index.html на сайте ИРЭ им. В.А. Котельникова РАН. На целесообразность подводить подобный итог через разумные промежутки времени нам не раз указывали читатели.
} 
На первом этапе это было получено частично в соавторстве с учеными других институтов и особенно, что было кардинально важно для исследований и дорого нам, В.И. Перелем.

a) В начале интенсивной пикосекундной оптической накачки тонкого слоя GaAs в нем возникает мощное стимулированное (усиленное спонтанное) излучение. Интегральное по спектру излучение релаксирует со временем $\geq 8$ пс, а его спектральные моды - с характерным временем $\geq 4$ пс. Излучение активно по отношению к вынужденному комбинационному рассеянию (ВКР).

б) Благодаря излучению возникает и поддерживается универсальная связь между температурой и плотностью неравновесных носителей заряда. Эта связь приводит к обратимому пикосекундному изменению плотности и температуры носителей. При фиксированном диаметре луча накачки после ее завершения носители релаксируют к универсальному пороговому их состоянию.

в) Излучение создает („выжигает“) обеднение заселенности электронов в реальном и в энергетическом пространствах. При этом оно наводит брэгговскую решетку заселенности в активной области слоя $\mathrm{GaAs}$, входящего в состав волноводной гетероструктуры $\mathrm{Al}_{x} \mathrm{Ga}_{1-x} \mathrm{As}-\mathrm{GaAs}-\mathrm{Al}_{x} \mathrm{Ga}_{1-x}$ As. Из-за этого появляется распределенная обратная связь (РОС), и накачанная область GaAs приобретает свойства РОС-лазера. Форма обеднения в энергетическом пространстве подобна форме спектра излучения.

г) Поле излучения возбуждает межзонные осцилляции электронов, создающие осцилляции обеднения заселенности. Амплитудофазочастотную характеристику последних определяют соответственно: (а) спектр излучения и его интерференционная картина; (б) ВКР спектральных мод излучения, синхронизирующее осцилляции электронов; (в) интенсивность излучения. Из-за осцилляций электронов модулируется с пикосекундными периодами интенсивность спектральных компонент излучения. Осциллирующее обеднение инверсной заселенности энергетических уровней на дне зоны проводимости транслируется, для восстановления детального равновесия, вверх по зоне проводимости. Трансляция с периодом, равным энергии продольного оптического (LO) фонона, распространяется до энергетического уровня, на который накачиваются электроны. Распределение электронов на участке от дна зоны проводимости до указанного уровня отклоняется от фермиевского и становится модулированным и синхронизованным. Соответственно возникает субтерагерцовая модуляция фундаментального поглощения света в слое $\mathrm{GaAs}$, a отсюда и интенсивности света при прохождении через этот слой. Мгновенная ширина спектра излучения становится лимитированной энергией LO-фонона. Модуляции заселенности энергетических уровней электронами, одновременно создаваемые в GaAs интенсивными пикосекундными импульсами внешнего и собственного стимулированного излучений, автосинхронизуются. ВКР спектральных мод излучения, возникающее при межзонных осцилляциях электронов, приводит к переключению мод. д) Энергетический транспорт накачанных носителей (ЭТН) в область экстремумов зон проводимости и валентной и вынужденная рекомбинация этих носителей находятся в динамическом равновесии, для поддержания которого излучение авторегулирует обеднение инверсной заселенности электронов. Таким образом ЭТН определяет интенсивность и длительность излучения. ЭТН и рекомбинация способствуют: замедлению залечивания отклонений от фермиевского распределения носителей; неэкранированию электрон-LOфононного взаимодействия; дефициту перенормировки ширины запрещенной зоны, вызываемой кулоновским взаимодействием носителей. Разогрев носителей заряда, создаваемый излучением, замедляет ЭТН, что в свою очередь замедляет возрастание и релаксацию излучения.

В прикладном отношении полученное представление о динамике излучения дало следующее. Открыло возможность обратимого с пикосекундной инерционностью изменения прозрачности и фотопроводимости GaAs. Предложило новые объяснения таких нестабильностей излучения, мешающих работе полупроводниковых лазеров, как автомодуляция интенсивности и спектра излучения (включая многомодовость), перемещение спектра в энергетическом пространстве, конкуренция и переключение спектральных мод. Объяснило влияние на излучение создаваемого им разогрева носителей и способы уменьшения этого влияния, включающие способы сокращения длительности и увеличения интенсивности излучения. Показало возможность усиления интенсивности излучения благодаря интенсификации ЭТН за счет BKP с эмиссией: (a) LO-фононов при накачке носителей на уровни с обедненной заселенностью; (б) плазмонов. Объяснило сужение спектра излучения при увеличении диаметра активной области. Как видно, созданное представление о вышеуказанной динамике актуально не только в фундаментальном, но и в прикладном аспектах. Часть перечисленных в нем результатов получена только эмпирически, и их теоретическое исследование могло бы привести, по нашему мнению, к предсказанию новых явлений.

\section{Финансирование работы}

Работа выполнена в рамках государственного задания.

\section{Конфликт интересов}

Авторы заявляют, что у них нет конфликта интересов.

\section{Список литературы}

[1] Н.Н. Агеева, И.Л. Броневой, Д.Н. Забегаев, А.Н. Кривоносов. ЖЭТФ, 143, 634 (2013).

[2] Н.Н. Агеева, И.Л. Броневой, Д.Н. Забегаев, А.Н. Кривоносов. ФТП, 53, 1471 (2019).

[3] Ю.Д. Калафати, В.А. Кокин. ЖЭТФ, 99, 1793 (1991).

[4] Н.Н. Агеева, И.Л. Броневой, Д.Н. Забегаев, А.Н. Кривоносов. ФТП, 54, 25 (2020). 
[5] Н.Н. Агеева, И.Л. Броневой, Д.Н. Забегаев, А.Н. Кривоносов. ЖЭТФ, 144, 227 (2013).

[6] Н.Н. Агеева, И.Л. Броневой, Д.Н. Забегаев, А.Н. Кривоносов. ФТП, 51, 594 (2017).

[7] С.Е. Кумеков, В.И. Перель. ЖЭТФ, 94, 346 (1988).

[8] Л.И. Глазман. ФТП, 17, 790 (1983).

[9] J.S. Blakemore. J. Appl. Phys., 53, R123 (1982).

[10] Н.Н. Агеева, И.Л. Броневой, А.Н. Кривоносов. ФТП, 35, 65 (2001).

[11] M. Combescot, P.J. Noziers. Phys. C, 5, 2369 (1972).

Редактор А.Н. Смирнов

\section{Influence on the duration of picosecond stimulated emission GaAs of charge carriers heating by this emission}

N.N. Ageeva, I.L. Bronevoi, D.N. Zabegaev,

A.N. Krivonosov

Kotel'nikov Institute

of Radioengeneering and Electronics,

Russian Academy of Sciences,

125009 Moscow, Russia

Abstract The real-time measured envelopes of high-power picosecond optical pumping and of the intrinsic stimulated picosecond emission of a thin layer GaAs are compared. The explanation for the excess of the emission duration over the pump duration is based on the negative feedback between the emission intensity and the heating of the carriers by this emission. The main parameter of the specified excess of the duration is the characteristic cooling time of charge carriers, which is slowed down due to heating of the carriers by emission. The influence of this time on the renormalization of the band gap due to the Coulomb interaction of carriers is also noted. The part of the conception of the picosecond dynamics of intense stimulated emission of GaAs, which has been obtained to present moment in our works with co-authors, is given in the Conclusion. 